\title{
DYNAMIC FOCUSING OF AN ELECTRON BEAM THROUGH A LONG PLASMA
}

C. O’Connell, F-J. Decker, M. J. Hogan, R. Iverson, P. Raimondi, R. H. Siemann, and D.

Walz

Stanford Linear Accelerator Center, Stanford, CA 94309

\author{
B. Blue, C. E. Clayton, C. Joshi, K. A. Marsh, W. B. Mori, and S. Wang \\ University of California, Los Angeles, CA 90095
}

T. Katsouleas, S. Lee, and P. Muggli

University of Southern California, Los Angeles, CA 90089

The focusing effects of a $1.4 \mathrm{~m}$ long, (0-2) x $10^{14} \mathrm{~cm}^{-3}$ plasma on a single $28.5 \mathrm{GeV}$ electron bunch are studied experimentally in the underdense or blowout regime, where the beam density is much greater than the plasma density. As the beam propagates through the plasma, the density of plasma electrons along the incoming bunch drops from the ambient density to zero leaving a pure ion channel for the bulk of the beam. Thus, from the head of the beam up to the point where all plasma electrons are blown out, each successive longitudinal slice of the bunch experiences a different focusing force due to the plasma ions. The time-changing focusing force results in a different number of betatron oscillations for each slice depending upon its location within the bunch. By using an electron beam that has a correlated energy spread, this time-dependent focusing of the electron bunch has been observed by measuring the beam spot size in the image plane of a magnetic energy-spectrometer placed at the plasma exit.

41.75.Lx, 41.75.Ht, 52.35.-g 


\section{INTRODUCTION}

Plasma based accelerators utilizing relativistic-propagating plasma waves (or "wakes") offer the potential of higher acceleration gradients and stronger focusing fields as compared to conventional RF methods. Both laser pulses and short electron bunches can be used as "drivers" for the excitation of relativistic plasma waves and both techniques have shown acceleration over short distances; typically over less than $1 \mathrm{~cm}$ (for lasers) to $10 \mathrm{~cm}$ (for electrons). ${ }^{12345}$ Experiments at the Stanford Linear Accelerator Center (SLAC) have been studying the longitudinal and transverse dynamics of electron-bunch drivers in a dense plasma $\left(n_{p}>10^{14} \mathrm{~cm}^{-3}\right)$ over an extended length of $1.4 \mathrm{~m}$, a length consistent with a prototype multi-GeV plasma-accelerator stage. The stable propagation of a tightly focused, short-pulsed and high-peak current electron drive bunch through such a stage is required for high-energy physics applications. A fundamental characteristic of such beam-plasma interaction is a time-dependent focusing force, which, in turn, gives rise to a rich oscillation behavior of the beam referred to as dynamic focusing. This paper presents measurements demonstrating the effects of dynamic focusing using the time integrated spot size of an electron bunch, which contains a correlated energy chirp, in the image plane of a magnetic energy-spectrometer placed at the plasma exit.

\section{PHYSICAL PICTURE}

When a short electron bunch propagates in a plasma, the plasma electrons are expelled by the space-charge force of the bunch. This motion of the plasma electrons forms a wake since the heavier ions are left behind. The optimal r.m.s. bunch length, $\sigma_{z}$, for driving a 
large-amplitude wake in a plasma of density $n_{p}$ is given by $\sigma_{z} \sim \pi c / 2 \omega_{p}$ where the plasma frequency $\omega_{p}$ is $\sqrt{\left(4 \pi n_{p} e^{2} / m_{e}\right)}$. The transverse fields of the wake impose a focusing force on the electron beam. In the case of a pre-formed ion channel of a given density, the beam can be "beta matched" coming into the plasma. When the beam is in this matched condition, it will propagate through the plasma without any variation in its transverse spot size, $\sigma_{r}$. In this experiment, the ion channel is not pre-formed and the plasma density is varied. The resulting envelope oscillations provide a useful diagnostic into the temporal dynamics of an electron bunch propagating through a plasma; in particular, the dynamic focusing within a single bunch as a result of the time-dependent focusing forces.

For the conditions of this experiment with $\sim 3.2 \mathrm{nC}$ of charge in a $\sigma_{z} \sim 0.65 \mathrm{~mm}$ electron bunch, the beam density, $n_{b}$, is much greater than the plasma densities. The situation of $n_{b} \gg n_{p}$ is called the "blowout" regime, where the density of plasma electrons is insufficient to neutralize the beam space charge and all the plasma electrons are blown out of the beam's path after a certain time.

The basic physics involved in dynamic focusing is as follows: the leading edge of the bunch expels electrons from the plasma leaving behind a column of massive (relatively immobile) ions. These ions then focus the beam via their space charge force. This focusing force rises from zero at the head of the bunch to the asymptotic value given by the pure ion-channel density (typically reached in the middle of the bunch for this 
experiment). The time necessary for all the plasma electrons to be expelled from the cross section of the bunch is referred to as the "blowout time." Thus each successive longitudinal "slice" in the front portion of the bunch experiences a different focusing force and consequently a different betatron oscillation frequency until blowout is reached. Elucidating the details of this dynamic behavior is the purpose of this paper.

\section{RELATED PRIOR MEASUREMENTS}

Previous papers have shown the clear oscillation of the projected or time-integrated beam spot size (betatron oscillations) due to the focusing effect of the plasma wakefield. ${ }^{67}$ As was presented in those analyses, the focusing force of the plasma increases as the plasma density increases, until the time-integrated beam spot size is a minimum at the image plane, which for those previous experiments was located $1 \mathrm{~m}$ downstream of the plasma exit. At higher densities, the focus will eventually be inside the plasma itself, and the time-integrated beam spot size increases at the image plane. At still higher densities, the first focus will be well inside the plasma and a second focus will be formed at the image plane. As this process continues the betatron motion becomes evident and the end result shows a focused beam at the image plane for multiple plasma densities. A simple envelope equation assuming a fully pre-formed, time-independent ion channel was found to match the data fairly well. The reason the envelope model and data agree is because the dominant contribution to the measured beam spot size was the bulk of the beam after blowout, meaning the dynamically focused part of the bunch was effectively washed out in the measured profile. 
In addition to demonstrating oscillation of the time-integrated beam size, there have been various experiments that have also shown time-resolved variation of the transverse beam size within a bunch using a streak camera. ${ }^{68}$ The limited dynamic range and timeresolution of the streak camera constrained the measurement of dynamic focusing to the plasma-lens case of a single minimum for these experiments. This paper expands on the work presented in Ref. 6 and significantly improves on the streak camera measurements as a result of a novel diagnostic method described in Section $\mathrm{V}$ of this paper. The improved time-resolution occurs by exploiting the correlated energy chirp within the bunch and the imaging properties of the spectrometer.

\section{NUMERICAL STUDIES}

Self-consistent simulations using the 3-D-cartesian (and 2-D-cylindrical) particle-in-cell

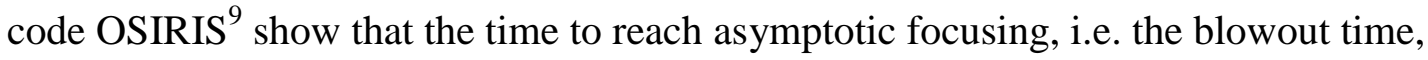
depends mainly on the beam's transverse field as determined by the beam density, $n_{b}$,

and is not substantially influenced by the plasma density (Fig.1). In this experiment, $n_{b}$ is not varied, so the time to blowout remains constant as the plasma density is varied. Since the strength of the focusing force on the beam does depend on the plasma density, the minima of the envelope oscillations will occur earlier in the bunch at higher plasma densities. Figure $2 \mathrm{a}$ illustrates the rise in the ion column density, $n_{i}$ (equal to the drop in the electron plasma density due to blowout) along the axis of an electron bunch for three different ambient plasma densities (solid lines). The temporal location of full blowout remains constant as indicated by the vertical dotted line. The arrows on the $n_{i}$ scale indicate the requisite ion densities needed to minimize the beam size at the plasma exit, 
which is the image plane for this analysis. From curve (i) of Fig. 2a, one sees that for some low plasma density the bunch will have just one minimum at a slice near the front of the bunch (indicated by the closed triangle). For some high density, illustrated in curve (iii) of Fig. 2a, three bunch slices, all prior to the blowout time, will be at a minimum at the plasma exit (indicated by the closed circles). The expected time-resolved spot size of the bunch at the plasma exit is sketched in Fig. $2 b$ where the three curves correspond to the three plasma densities of Fig. 2a and the location of the symbols in time are the same as in Fig. 2a.

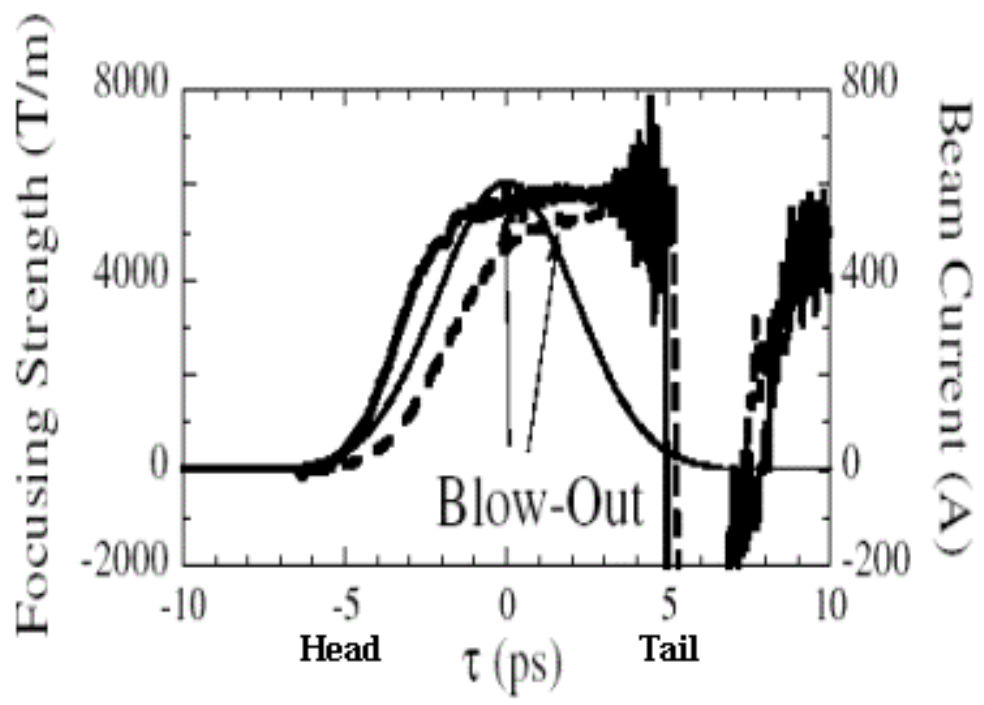


FIG. 1. Transverse focusing strength of the wake excited by an electron bunch (thin line) at a high bunch density (continuous line) and a low bunch density (dashed line) in a plasma with a constant plasma density, $n_{\mathrm{p}}=2 \times 10^{14} \mathrm{~cm}^{-3}$, as obtained from particle-in-cell computer simulations.

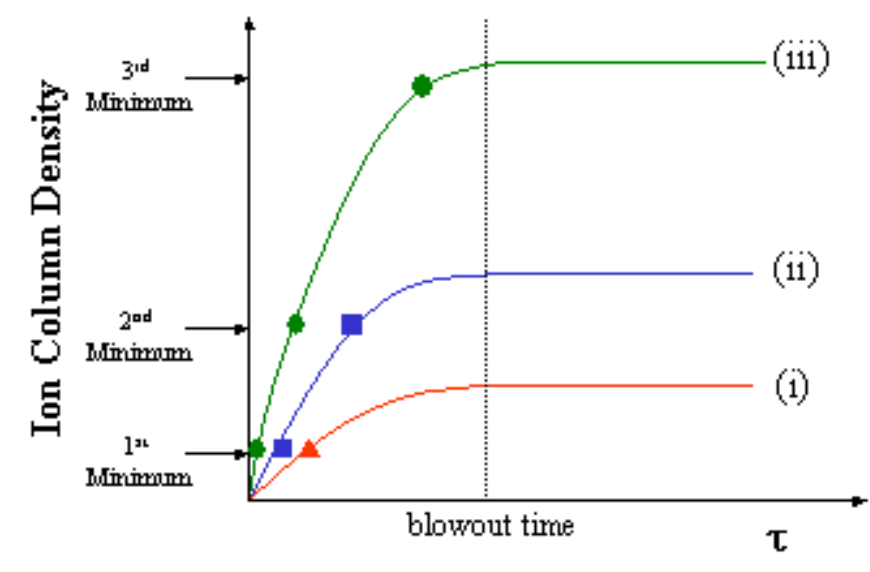

(a)
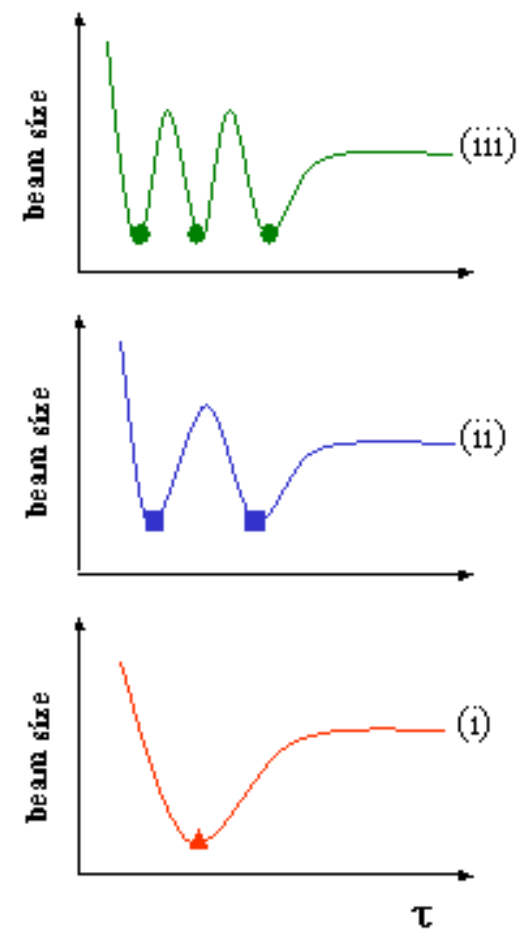

(b) 
FIG. 2. (a) A schematic representation of the ion column density along the bunch in time at several ambient plasma densities. The lowest density is line (i) and the highest density is line (iii). The three arrows along the ion column density axis indicate the density at which the beam is a minimum at the plasma exit. The lowest density has only one focus, while the highest density undergoes three minima. (b) Pictorial representations for the expected beam sizes at the plasma exit for the three densities given in (a).

Animated results from a numerical model of this time-dependent focusing force on the beam spot size at the plasma exit are presented in Video 1. The video assumes a perfect gaussian distribution of charge along the transverse and longitudinal components. Figure 3 shows a typical frame from approximately midway through the video, which shows time flowing from the top of the image to the bottom versus the transverse component, centered along horizontal axis. For every frame of Video 1 , the ion column density $n_{i}$ is being linearly ramped from zero at the leading edge to the ambient plasma density midway through the bunch. The net focusing force at any given location along the bunch is proportional to $\sqrt{n_{i}}$. Each successive frame then incrementally increases the ambient plasma density from $1 \times 10^{12} \mathrm{~cm}^{-3}$ to a maximal value of $2 \times 10^{14} \mathrm{~cm}^{-3}$ in 151 steps. It should be noted that the focusing force is assumed to be linearly proportional to the radius throughout the bunch. This approximation is only strictly valid for the majority of the bunch that is in the blowout regime. ${ }^{6}$ Some characteristics to notice are the increase in the amount of betatron oscillations within the bunch as the ambient plasma density increases, as well as the temporal migration of the minima associated with these 
oscillations to times earlier in the bunch as the density increases. Also note that the latter half of the bunch, the part of the beam that is in blowout, experiences a constant focusing force at a given density.

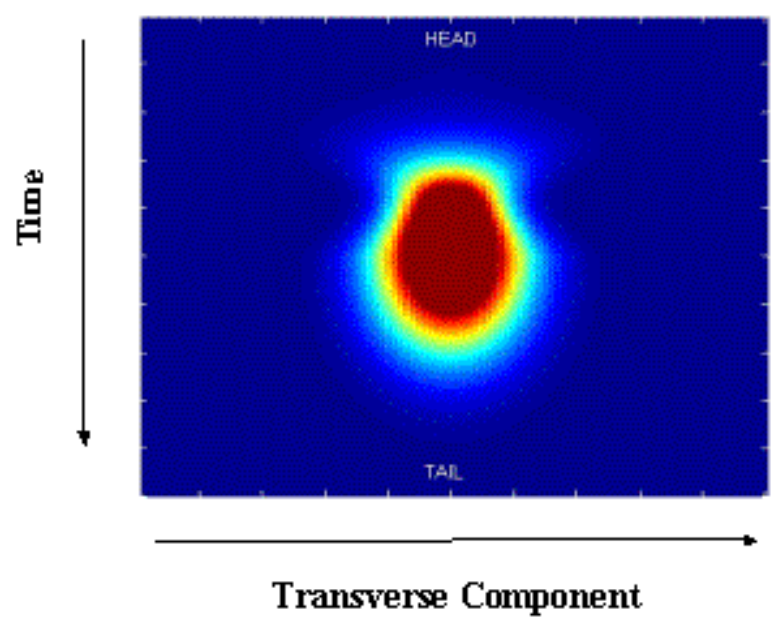

FIG. 3. (Video) A single frame from Video 1 showing the beam density versus time and transverse position as it exits the plasma, where time flows from the top of the image to the bottom and the horizontal axis is the transverse component of the beam centered along the middle of the axis. In each frame, the front half of the bunch experiences an increasing focusing force until blowout midway through the bunch at which point the focusing force remains constant. The ambient plasma density is increased for each subsequent density.

\section{EXPERIMENTAL METHOD}

The experiment described in this paper, known as E-162, is ongoing at SLAC and uses the $28.5 \mathrm{GeV}$ electron beam at the Final Focus Test Beam (FFTB) facility. Figure 4 illustrates the primary features of the experimental setup. The incoming bunch is $\sim .65$ 
$\mathrm{mm}$ long and has a full-width energy spread of $1.8 \%$. The correlated energy spread of the bunch is determined by the wakefields in the linac and where the bunch rides the rf wave. An ultraviolet (UV) laser pulse ionizes the lithium vapor prior to the bunch's arrival and the resulting plasma density is linearly proportional to the incident energy of the ionizing laser. The lithium plasma, $1.4 \mathrm{~m}$ in length, ranges in density from $(0-2) \times 10^{14} \mathrm{~cm}^{-3}$. Optical transition radiators $(\mathrm{OTR})$ located $\approx 1 \mathrm{~m}$ upstream and $\approx 1 \mathrm{~m}$ downstream of the plasma image the beam before entering and after exiting the plasma. A magnetic imaging energy-spectrometer images the electron beam from the plasma exit onto a Cherenkov radiator, 25 meters downstream of the plasma. This imaging spectrometer, consisting of six quadrupoles and a large dipole, has a magnification of 3, to within $10 \%$, in both the horizontal and vertical dimensions. The dispersion $\eta$ at the spectrometer image plane is $\approx 10 \mathrm{~cm}$ or $\sim 300 \mathrm{MeV} / \mathrm{mm}$. See Table 1 for the typical beam and plasma parameters.

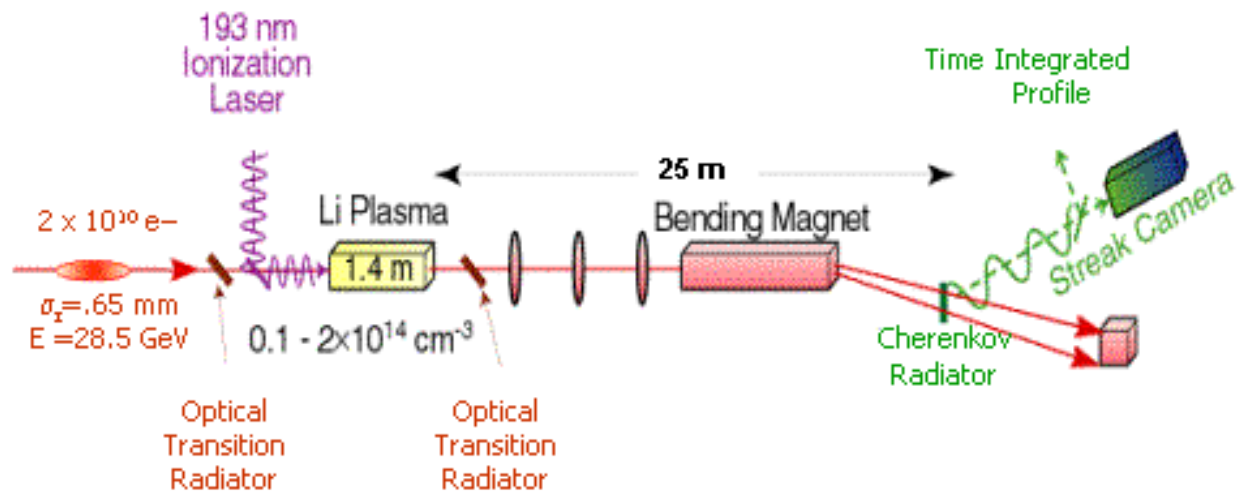

FIG. 4. Schematic of E-162 experiment layout. The diagram is not to scale. 


\begin{tabular}{|c|c|c|}
\hline Number of e- per bunch & $N$ & $2 \times 10^{10}$ \\
\hline Bunch Energy & $E, \gamma$ & $28.5 \mathrm{GeV}, 5.6 \times 10^{4}$ \\
\hline Bunch Length & $\sigma_{z}$ & $.65 \mathrm{~mm}$ \\
\hline Full-Width Energy Spread & & $.8 \%$ \\
\hline $\begin{array}{c}\text { Uncorrelated Energy Spread } \\
\text { a }\end{array}$ & & $25 \mu \mathrm{m}, 20 \mu \mathrm{m}$ \\
\hline $\begin{array}{c}\text { Transverse Beam Size at Plasma } \\
\text { Entrance }\end{array}$ & $\sigma_{x}, \sigma_{y}$ & $165 \mu \mathrm{m}, 765 \mu \mathrm{m}$ \\
\hline $\begin{array}{c}\text { Transverse Beam Size at Cherenkov } \\
\text { Radiator without Plasma }\end{array}$ & $\sigma_{x}, \sigma_{y}$ & $26,7 \times 10^{-5} \mathrm{~m}^{*} \mathrm{rad}$ \\
\hline $\begin{array}{c}\text { Normalized Emittances at Plasma } \\
\text { Entrance }\end{array}$ & $\varepsilon_{x}, \varepsilon_{y}$ & $(0-2) \times 10^{14} \mathrm{~cm}^{-3}$ \\
\hline $\begin{array}{c}\text { Plasma Density } \\
\text { Plasma Length }\end{array}$ & $n_{p}$ & $1.4 \mathrm{~m}$ \\
\hline
\end{tabular}

\section{TABLE 1. Typical beam and plasma parameters.}

To determine the proportionality constant between the plasma density and the measured incident UV laser energy, the densities for which the beam is focused to a minimum at the plasma exit are calculated from the beam envelope model and compared to the experimental data. ${ }^{6}$ The initial Twiss parameters at the plasma entrance (which give the emittance, initial spot size and beam-convergence needed for the envelope model) are obtained for the horizontal plane by the standard three-screen technique using the laseroff profiles from the upstream and downstream OTR images and the Cherenkov image.

\footnotetext{
${ }^{a}$ Energy Spread at a Given Time
} 
In the laser-on beam envelope calculations, the plasma is modeled as a thick quadrupole that focuses in both planes. The single free parameter, the constant of proportionality between the UV energy and plasma density, is obtained by fitting the model predictions to experimental spot-size data when the UV energy is varied.

The magnetic energy-spectrometer images the beam at the plasma exit onto a thin piece of transparent aerogel. ${ }^{10}$ The resulting Cherenkov light is then collected and re-imaged by an all-reflective optical transport system to a streak camera located 15 meters from the beamline. A reflective-optics system, having no refractive optical elements, is achromatic and the very-broadband Cherenkov light is re-imaged with no color or temporal aberrations. Two large field mirrors are used in the system for relay imaging, resulting in a large $(\approx 7.5 \mathrm{~mm})$ field-of-view of the Cherenkov plane with high transmission and no vignetting. Since many of the mirrors are spherical, off-axis reflections introduce astigmatism. Therefore, each spherical mirror is paired with an identical mirror at an identical (but opposite) tilt-angle such that the astigmatisms cancel each other to lowest order. The resulting spatial resolution of the entire Cherenkovimaging system is approximately $100 \mu \mathrm{m}$. With the $\sim 300 \mathrm{MeV} / \mathrm{mm}$ Cherenkov-plane dispersion, this corresponds to an energy resolution of $\sim 30 \mathrm{MeV}$ or about $0.1 \%$. A beam splitter samples a fraction of the transported light to provide a time-integrated image of the beam in the Cherenkov plane, recorded with a 12-bit charge coupled device (CCD) camera. The remainder of the light is then split and directed to the streak camera along two paths or arms. The horizontal arm is delayed $\sim 20 \mathrm{psec}$, rotated $90^{\circ}$ and recombined with the vertical arm. The final spherical mirror images the Cherenkov radiator through 
both arms onto the slit of the streak camera. This setup allows observation of the bunch's longitudinal dynamics along both the horizontal and vertical directions. The streak camera has time resolution of $\approx 1$ psec. $^{6}$

Although the streak camera has sufficient resolution for tracking energy changes within the bunch, it is not the best tool for studying detailed transverse dynamics. The requirement of having a narrow slit (necessary for obtaining time resolution) limits the transverse field-of-view at the object (Cherenkov) plane. This is illustrated in Fig. 5 where the field-of-view of the streak camera is indicated by the horizontal and vertical input slits superimposed on a time-integrated image of the beam at the Cherenkov plane. When analyzing the horizontal dimension, most of the beam's image is lost outside the slit as is clear from Fig. 5. Also, although the vertical slit is aligned with the center of the beam, much of the envelope modulation along the outer edge of the image is not captured. Although the streak camera has some limitations, it is necessary in determining the time correlated energy distribution of the incoming electron bunch. Plotted on Fig. 6 are the mean vertical positions of 24 time-slices from the streak camera image data vs. the relative time of the slices, with $\tau=0$ defined as the slice with the highest current (the gaussian current profile of the bunch is also shown as the solid line in Fig. 6). The energy chirp shown in Fig. 6 is roughly linear from $\sim-5$ psec to +3 psec where the head of the bunch is more energetic than the tail. Comparing Figs. 5 and 6, one sees that the linear portion of the chirp, coupled with the dispersion generated by the dipoles in the imaging spectrometer, can make the time-integrated Cherenkov profiles a temporal diagnostic. Since the vertical position at the Cherenkov radiator is dominated by the 
energy dispersion (due to the imaging spectrometer), the vertical dimension of the timeintegrated Cherenkov images effectively functions as a temporal axis that is approximately linear in the corresponding linear portion of the chirp. The optical resolution along the vertical dimension of the Cherenkov images limits the effective temporal resolution to $\sim 400 \mathrm{fsec}$ in the region where the energy chirp is linear. By capturing the entire spatial extent of the bunch, the time-integrated Cherenkov profiles provide a complementary - and in the case of transverse dynamics, a superior - temporal diagnostic to the streak camera.

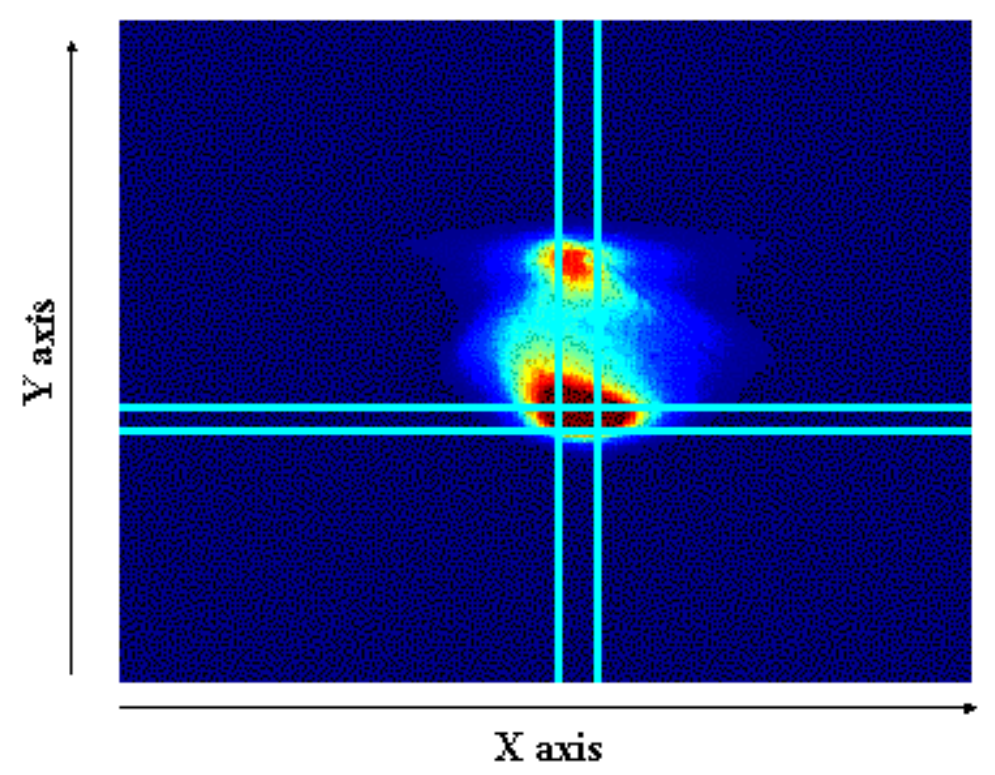


FIG. 5. Typical time-integrated Cherenkov image with superimposed slit locations of the streak camera in the horizontal and vertical direction.

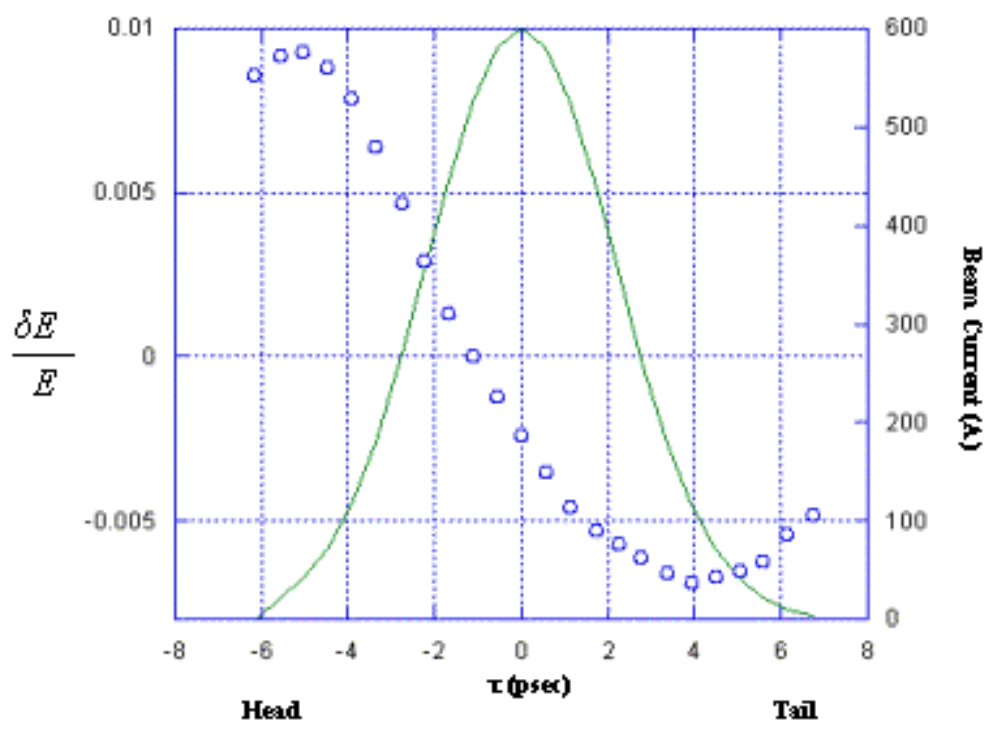

FIG. 6. The measured correlated energy spread of the beam (open blue circles) and the current distribution (green solid line).

\section{EXPERIMENTAL DATA}

Experimental time-integrated Cherenkov image-data is shown in Video 2. The data set that forms the movie originates from a run of two hundred events, taken at $1 \mathrm{~Hz}$. Fifty laser-off shots, taken every forth event, are not included here. The incident UV laser energy was progressively increased over the 150 events in the video. Some behavior to notice are the modulations of the beam envelope along the bunch (i.e., in the vertical direction) as well as the minima of the horizontal slice sizes moving earlier (upward) within the bunch as density increases. These features, clearly seen in the data, were 
previously discussed as traits associated with dynamic focusing in Secs. II-IV of this paper and illustrated in Fig. 2 and 3.

Figure 7 shows four frames selected from Video 2 demonstrating the characteristics inherent in dynamic focusing. Figure 7 a (frame 47) shows the expected behavior at low plasma density, where the head of the bunch is unfocused and the latter portion of the bunch is weakly focused. In Fig. 7b (frame 61), the minimum of the beam envelope is beginning to move to earlier time, as is indicated by the concentration of intensity (the patch of red) moving upwards, away from the tail. In Fig. 7c (frame 78), the beam envelope is modulated such that the beam is focused near the head and tail but defocused in the middle of the bunch. Finally Fig. 7d (frame 93) shows the second minimum beginning to move up towards the head of the bunch.
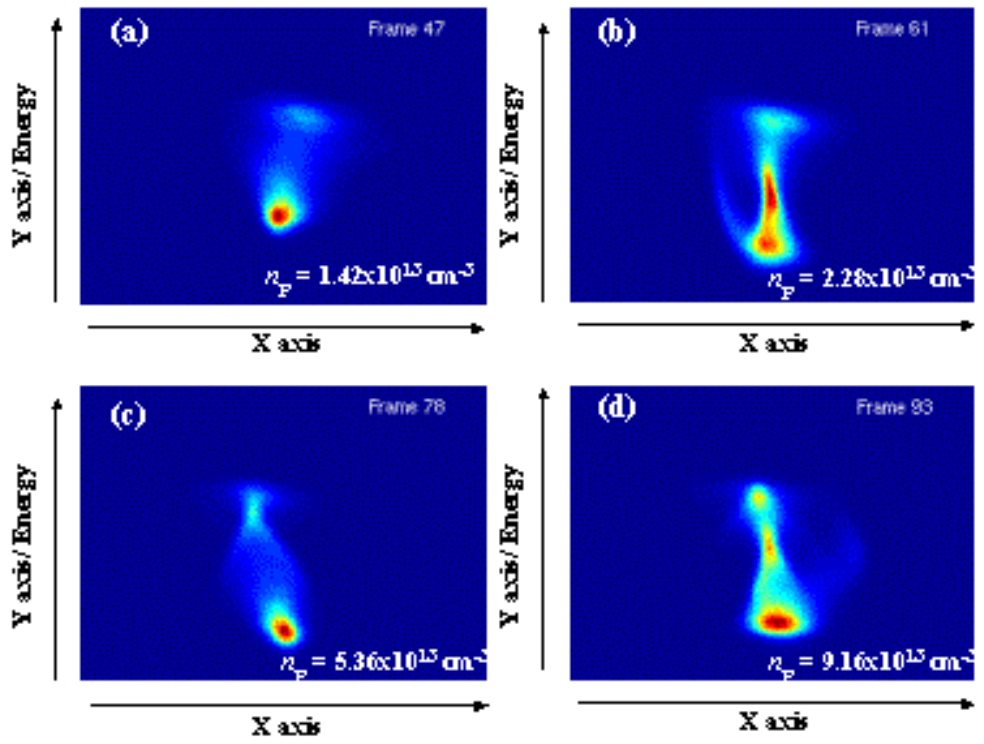

FIG. 7. (Video) Selected Frames from Video 2 with explanations given in the text. 
Video 2 qualitatively illustrates dynamic focusing of the bunch as the plasma density is increased. To quantify the results, the Cherenkov images from Video 2 are sliced horizontally, such that each slice represents a small section in time. The region of the bunch where the energy chirp is roughly linear, was sectioned into $\sim 0.7$ psec-wide slices. It should be noted the results are not sensitive to the exact choice of this slice width. The data within each of the slices was summed vertically and fit to a gaussian in order to extract the characteristic horizontal r.m.s. width of each slice. Figure 8 shows an example of this analysis for two such slices.

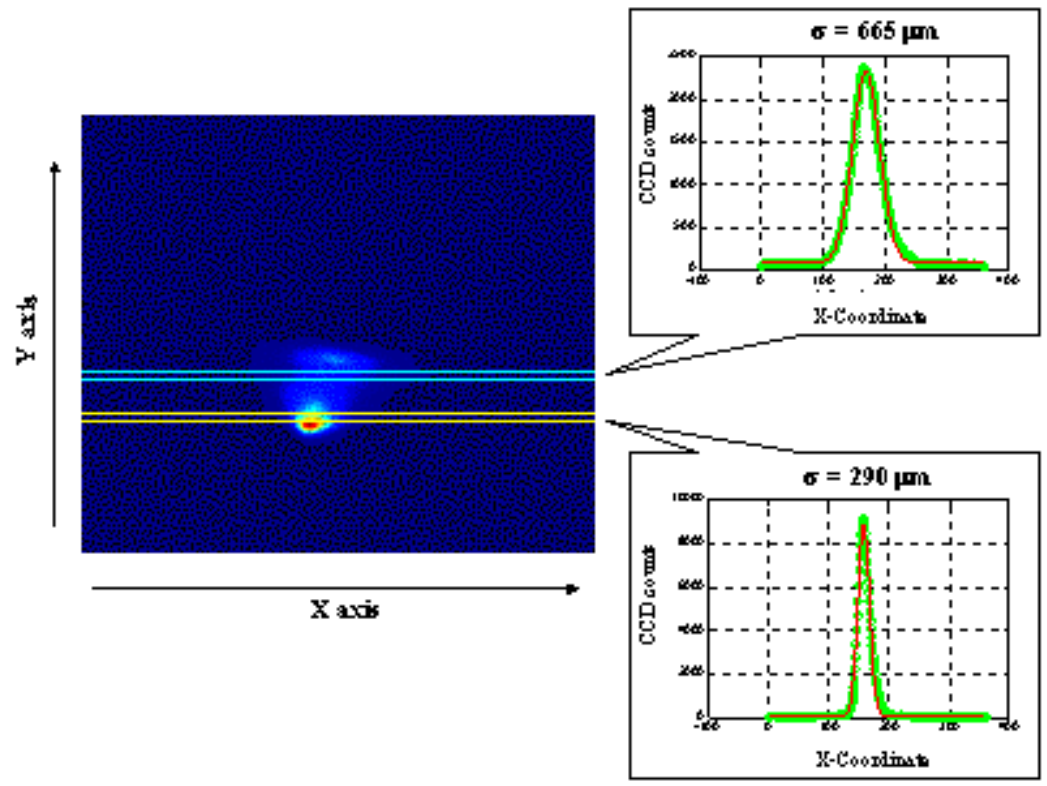

FIG. 8. Gaussian fits for a given Cherenkov image. For the graphs on the right, the green circles indicate the data and the Gaussian fit is the solid magenta line.

From Fig. 8, one can imagine how these slice profiles should vary along the bunch if the chirp were perfectly linear. The very top of the Cherenkov image (not seen in this colormap) would be the very front of the bunch and thus unaffected by the plasma. As 
the gaussian fit moves down toward the middle of the Cherenkov image, the width of the gaussian fit would oscillate, with the number of oscillations depending on the plasma density at which the Cherenkov image was taken. Still further down, into the bottom half of the Cherenkov image, the constant focusing force along the bunch due to blowout would result in a fit of some constant size, dependent on the plasma density for that image.

The results of the fitting analysis for the sectioned Cherenkov image are shown in Fig. 9 and repeated for three different plasma densities (three Cherenkov images) and, for comparison, the beam spot size with no plasma. We see that for each of these densities, the r.m.s. transverse size does indeed change along the bunch. Moreover, the location of transverse features, such as the minima, moves to times earlier in the bunch (as indicated by the solid black arrows in Figs. 9b-9d) with increasing density, as expected, due to the increase of the focusing force throughout the bunch at higher densities. In Fig. 9d, another minimum is beginning to form in the tail of the bunch as shown by the wider green arrow. Note the similarity of this data with the pictorial representations of Fig. 2. 
(a)

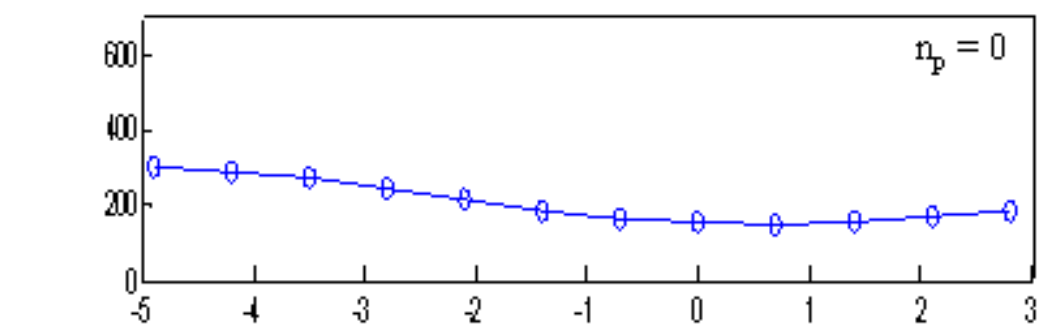

(b)

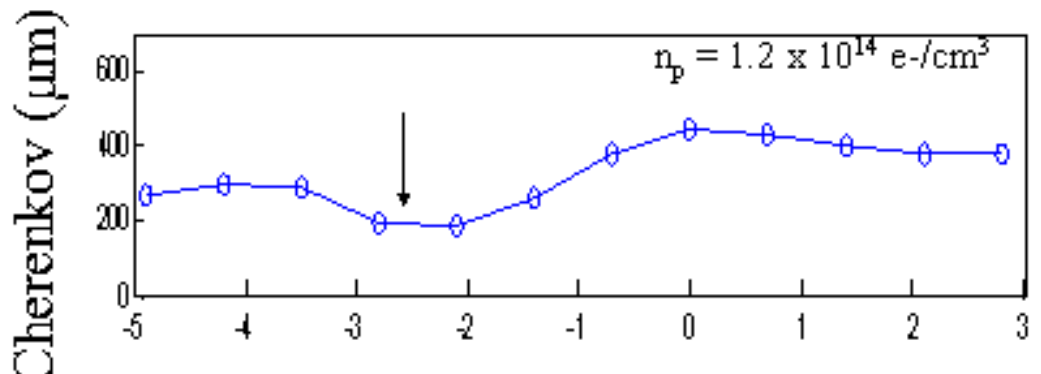

(c)

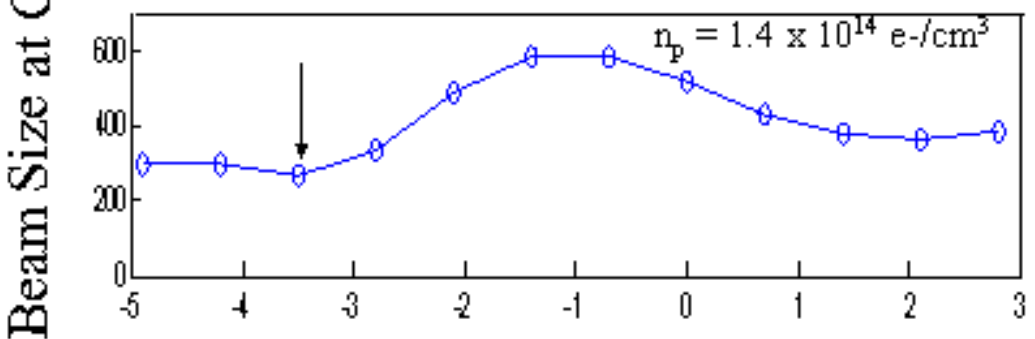

(d)

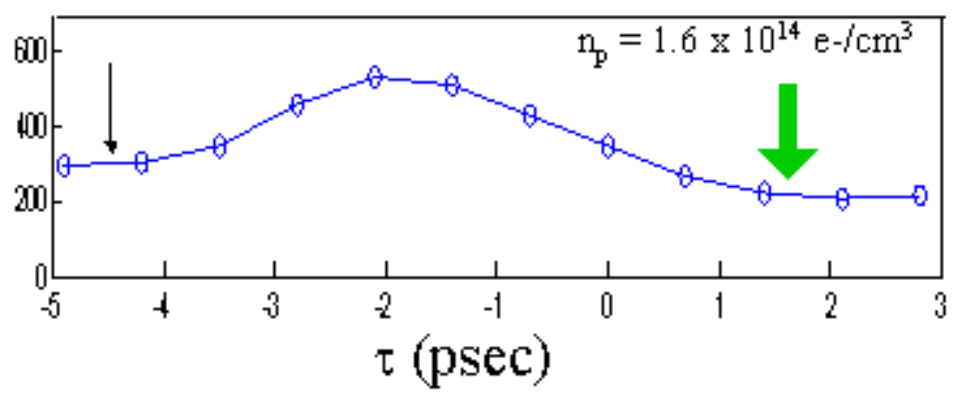

FIG. 9 Results of the fitting analysis of sectioned Cherenkov-plane images (see text). The horizontal axis is time and the vertical axis is the beam size at the Cherenkov radiator. The graphs increase in density from top to bottom. The minimum, denoted by the black arrows, moves earlier in time as the density increases. Also graph (d), the highest density, shows a second minimum (seen along the entire portion of the beam in blowout) beginning to form, denoted by the larger green arrow. 
We can achieve additional insight into dynamic focusing by re-plotting the data in Figs. 9b-9d and including a wide range of plasma densities. Figures 10a-101 show twelve plots, where each plot gives the density dependence of the transverse spot size for each of the 0.7 psec-wide slices of sectioned Cherenkov images. Here, the twelve horizontal axes are the same and give the ambient plasma density. The vertical axes display the spot size for the labeled slice (e.g., $\tau=-4.9$ psec in Fig. 10a is the first slice) of the time-integrated Cherenkov image. Thus, each plot represents a section in time, where time progresses from left to right, then top to bottom. However, as discussed previously, the time scale is only truly linear in the central region. We see in Fig. 10 that the number of betatron oscillations within the bunch increases towards the back of the bunch (Figs. 10a-10g) but only up to the blowout time occurring approximately at the plot labeled $\tau=0$ psec (Fig. 10h). Clearly, each successive slice of the bunch, from $\tau=-4.9 \operatorname{psec}$ to $\tau=0$, is experiencing a stronger effective focusing force than the slice prior to it. Conversely, the ambient plasma density needed to reach any given minimum decreases with time along the bunch. The locations of the minima are both slice- and density-dependent. 


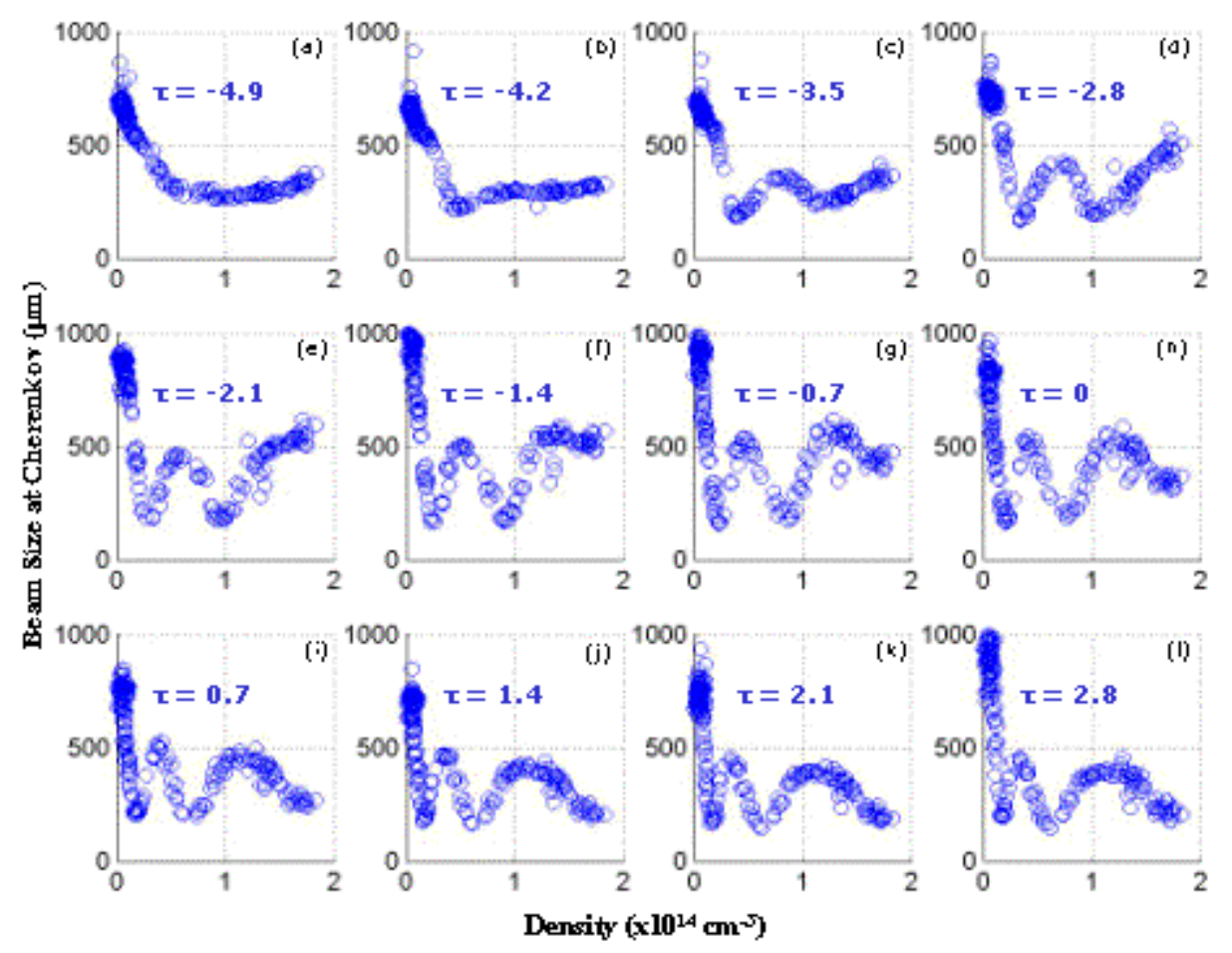

FIG. 10. The spot sizes of individual Cherenkov time slices $(\operatorname{read} L-R, T-B)$ from the analysis of Fig. 9 for ambient plasma densities up to $1.8 \times 10^{14} \mathrm{~cm}^{-3}$. Graph (a) shows the weak focusing force, which dominates the first head slice. Beginning at graph (b) the slices are in the linear portion of the chirp. Graphs (i)-(l) are in the blowout regime, since the focusing force is no longer changing.

The density and time location of the first and second minima from Fig. 10 are summarized in Fig. 11. Plotted here are the densities at which each slice has been focused once (red circles) and twice (blue squares). From these curves, it appears that the dynamic behavior stops at around the center of the bunch, as expected from the PIC simulation results of Fig. 1. Figure 11 also shows that, after blowout when the transverse forces are due to a simple ion column, there are specific densities for which all the later slices of the beam will be focused. This implies, for example, that the evolution of the 
envelope of a witness or accelerating bunch placed in this blowout region should be predictable from the optical properties of an ion column - a necessary requirement for applications of such plasmas to staged accelerators.

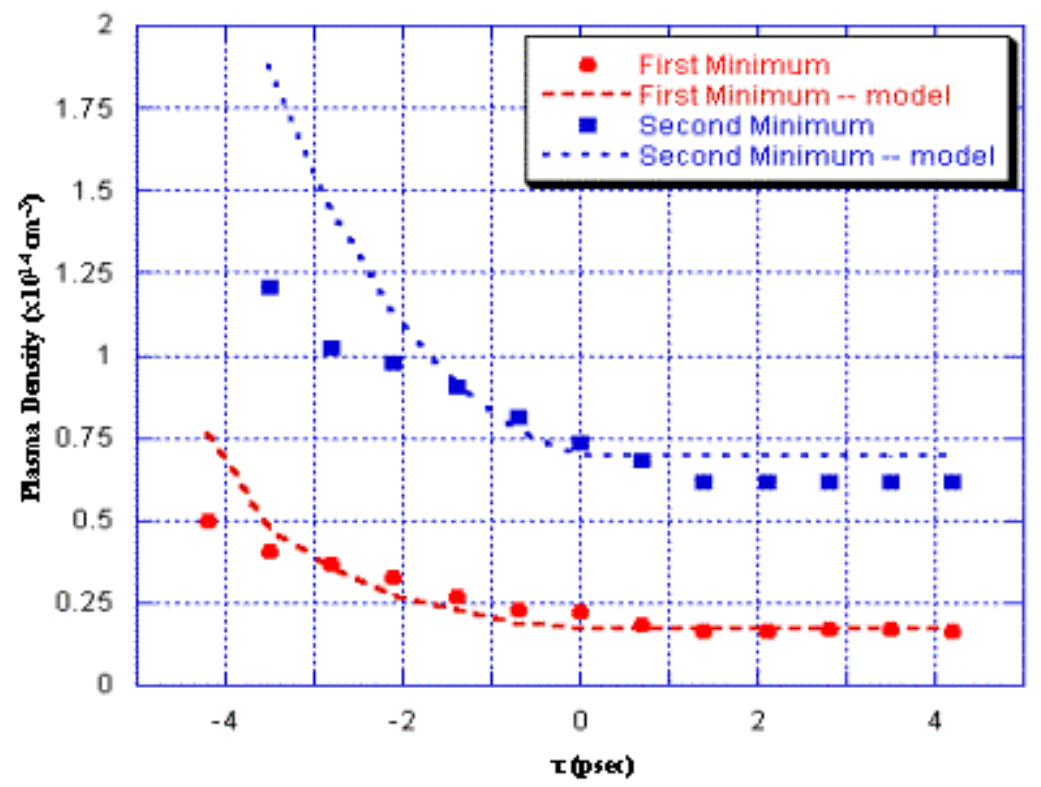

FIG. 11. The density location of the first two minima of each slice of Fig. 10 versus the temporal position of the slice. The red circles represent the movement of the first minimum in time (the red dashed line indicates the model's predictions for the first minimum) and the blue squares represent the second minimum (the blue dotted line indicates the model's predictions for the second minimum).

Also shown in Fig. 11 are results from the same model used to generate Video 1. The dashed red line follows the time and density required to arrive at the first focus (within the bunch) at the plasma exit. Similarly, the dotted blue line follows the time and density required for the second focus. The discrepancy between the model and the data is due 
mostly to the mixing of time and energy at head of the bunch as measured at the Cherenkov plane. This issue is discussed further in the next section.

\section{LIMITATIONS}

Although the time-integrated Cherenkov images are not limited by slits and provide better time resolution than the streak camera, they can only be utilized to understand the middle of the bunch. Since the Cherenkov image is actually spread out in energy and not time, the curvature or "fold-over" of the chirp as is seen in Fig. 6 will undermine the accuracy of the head and tail measurements. For example, the first slice of the Cherenkov image will include both the $-6 \pm 0.5 \mathrm{psec}$ and the $-5 \pm 0.5 \mathrm{psec}$ ranges or sections of the bunch. Since the latter sections have more particles that experience a weak-focusing force created by the initial section, those will dominate the "head" of the sliced Cherenkov image. This could cause the points representing the head of the bunch to be plotted too low on the density axis of Fig. 11. The same fold-over effects are seen in the tail of the bunch. This, together with the rapid and density-dependent change of the transverse forces at the tail of the bunch (Fig. 1) make the tail of the bunch too varied to be analyzed.

In addition, de-acceleration and acceleration will change the internal energy spectrum and cause some spatial mixing of the time slices in the integrated images. The full width correlated energy spread of the bunch is marginally perturbed when it passes through the plasma, however there can be significant internal energy changes resulting from the plasma. One effect is energy loss in the body of the beam, which can be interpreted as an 
effective time shift. Along the linear chirp in the center of the beam, the maximum time shift at the highest density would be $\sim 0.6 \mathrm{psec}$. Also only particles in the tail of the bunch are accelerated by the plasma wakefield. This is an additional complication in the measurement of dynamic focusing in the tail, which is already dominated by the curvature of the chirp. At the highest densities, tail particles can gain sufficient energy such that they will overlap with particles in the core, causing an effective shift in measured "time." Slices in the back half of the bunch with $\tau>0$ should only be affected. However, there are a relatively small number of accelerated particles and therefore its impact is negligible. Consequently, the Cherenkov images only accurately illustrate transverse dynamics for the middle of the bunch where the energy-time correlation is approximately linear and the time-dependent effects, which are the subject of this paper, are dominant.

\section{CONCLUSIONS}

In summary, the experimental results, using Cherenkov images as a temporal diagnostic, clearly illustrate the time dependent variation of the beam envelope size. The most general features of dynamic focusing include modulation of the beam envelope and beam spot-size minima that move earlier in time as the plasma density increases. The latter is in good agreement with predictions of a numerical model. This time dependent variation of the beam envelope is observed with a temporal resolution of approximately $400 \mathrm{fsec}$.

Any plasma accelerator will experience both longitudinal and transverse effects, but this work indicates that the transverse effects are well understood and do not present an 
obstacle for a properly placed witness bunch to utilize the longitudinal acceleration effects.

\section{Acknowledgements}

This work is supported by U.S. DoE Grants No. DE-FG03-92ER40745, No. DE-AC0376SF00515, No. DE-FG03-98DP00211, No. DE-FG03-92ER40727 and No. DE-AC-0376SF0098, and NSF Grants No. ECS-9632735 and No. DMS-9722121. We would like to thank Dr. Peter Tsou of JPL for providing the aerogel and Karl Erickson for assistance in collecting data.

\footnotetext{
1 A. Modena et al., Nature (London) 37, 606 (1995).

${ }^{2}$ C. I. Moore, A. Ting, K. Krushelnick, E. Esarey, R. F. Hubbard, B. Hafizi, H. R.
}

Burris, C. Manka, and P. Sprangle, Phys. Rev. Lett. 79, 3909 (1997).

${ }^{3}$ R. Wagner, S.-Y. Chen, A. Maksimchuk, and D. Umstadter, Phys. Rev. Lett. 78, 3125 (1997).

${ }^{4}$ D. F. Gordon et al., Phys. Rev. Lett. 80, 2133 (1998).

${ }^{5}$ N. Barov, J. B. Rosenweig, M. E. Conde, W. Gai, and J. G. Power, Phys. Rev. ST Accel. Beams 3, 011301 (2000).

${ }^{6}$ C. E. Clayton et al., Phys. Rev. Lett. 88, 154801 (2002).

${ }^{7}$ C. Joshi et al., Physics of Plasmas 9, 1845 (2002).

8 N. Barov, M. E. Conde, W. Gai, and J. B. Rosenzweig, Phys. Rev. Lett. 80, 81 (1998). 
9 R. G. Hemker, F. S. Tsung, V. K. Decyk, W. B. Mori, S. Lee, and T. Katsouleas, Particle Accelerator Conference, New York, 3672, (1999), edited by A. U. Luccio

${ }^{10}$ R. Assmann et al., Particle Accelerator Conference, New York, 330, (1999), edited by A. U. Luccio 